\title{
Postpartum stress fracture of bilateral tibia: A case report
}

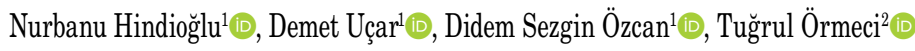 \\ ${ }^{1}$ Department of Physical Medicine and Rehabilitation, Istanbul Medipol University School of Medicine, Istanbul, Turkey \\ ${ }^{2}$ Department of Radiology, Istanbul Medipol University School of Medicine, Istanbul, Turkey
}

Received: June 22, 2019 Accepted: December 12, 2019 Published online: May 25, 2021

\begin{abstract}
Stress fractures are overuse injuries of the bone characterized by a magnetic resonance imaging (MRI) finding of the fracture line with bone marrow edema. Clinical findings are tenderness and persistent pain. It is usually related to repetitive stresses. A 25-year-old woman was admitted with bilateral severe knee pain. She was in the postpartum period and the complaints started three days after the beginning of 2-h daily walk. The initial plain radiograph showed no abnormality, while MRI demonstrated bilateral incomplete fracture line at the proximal tibia. The patient had accompanying vitamin D deficiency and osteopenia, diagnosed with pregnancy-related osteopenia and stress fracture of bilateral proximal tibia. We advised cessation of weight bearing, resting, and supplementation of calcium and vitamin D. This report highlights that, in the postpartum period, persistent pain may indicate bone lesions such as stress fractures, particularly developing shortly after a vigorous physical activity. Detailed physical examination and further investigations are necessary to detect these fractures and risk factors.
\end{abstract}

Keywords: Postpartum period, stress fracture, tibia.

Stress fractures include bone insufficiency and fatigue which can cause pain and dysfunction depending on the site of the involvement. Insufficiency fractures are usually caused by normal stress applied to the abnormal bone structure. Fatigue fractures are usually seen in athletes as a result of excessive stress and cycling load for prolonged periods on normal bones. ${ }^{[1,2]}$ The incidence of stress fractures is less than $1 \%$ in the general population. ${ }^{[3]}$ Lower extremities are usually involved in these patients and the second, third, and fourth metatarsal bones, tibia, pelvis, fibula, and humerus are the commonly affected areas. ${ }^{[4]}$

Risk factors for stress fractures are osteopenia or osteoporosis, substantial increase in intensity or volume of activity, decreased muscle strength or endurance, dietary disorders (i.e., insufficient calcium intake, vitamin D deficiency, or eating disorders), family history of osteopenia or osteoporosis, female sex, low body mass index, prolonged glucocorticoid use, previous stress fractures, and older age. ${ }^{[5,6]}$ Pregnancy and lactation period may also lead to decreased bone mineral density, causing postpartum stress fractures. ${ }^{[7]}$

In the literature, there are several case reports showing stress fractures of the sacrum, ${ }^{[8]}$ pubic bone, ${ }^{[9]}$ proximal femoral diaphysis, neck of femur and subcapital hip region, ${ }^{[10]}$ rib, ${ }^{[11]}$ and vertebrae related to postpartum osteoporosis. In this report, we present a postpartum woman with bilateral stress fracture of tibia. To our best of knowledge, there is no other case report of postpartum stress fracture with bilateral tibial involvement and this is the first case reported in the literature.

\section{CASE REPORT}

A 25-year-old woman was admitted to our physical medicine and rehabilitation outpatient clinic with bilateral severe knee pain for one week. The pain was localized and sharp in nature. The patient gave birth

Corresponding author: Nurbanu Hindioğlu, MD. İstanbul Medipol Üniversitesi Fizik Tedavi ve Rehabilitasyon Anabilim Dalı, 34214 Bağcllar, İstanbul, Türkiye. e-mail: nurbanuhindioglu@gmail.com 
a month ago and the symptoms occurred three days after starting 2-h daily walks. The initial pain score was 3 as assessed by the Visual Analog Scale (VAS) and progressed to 8 in the following days. The severity of pain increased with walking and bending of the knees. The patient had no history of trauma, previous fractures, systemic or metabolic diseases, and eating disorders. She was in lactation period and did not take any medication including supplementation of vitamin $\mathrm{D}$ and/or calcium; however, dietary calcium intake of the patient was found to be within normal ranges. On physical examination, no pathologies including swelling, redness, or any scar were detected. The ranges of motion of both knees were preserved, but painful through the flexion. The McMurray test result was positive for both knees; however, the other tests for meniscus and ligamentous injury were negative. Neurological examination findings were also normal. Deep tendon reflexes were normoactive and no motor, neither sensorial loss was detected. Plain radiographs of both knees (anteroposterior and lateral projections) were taken for the initial evaluation. The joint spaces were normal, and no fracture nor sclerotic line was detected. For a more detailed investigation, bilateral magnetic resonance imaging (MRI) of the knees were taken which showed an incomplete fracture line at the medial proximal tibia and bone marrow edema throughout the proximal tibia bilaterally (Figure 1). The MRI also showed that bilateral anterior cruciate ligaments were partially ruptured. We also performed bone single-photon emission computed tomography/ computed tomography (SPECT/CT) and whole-body bone scintigraphy. The investigations were interpreted as a benign process with an increased activity at the level of medial tuberosities of tibias bilaterally (Figure 2). The laboratory tests revealed vitamin $\mathrm{D}$ deficiency, $(8 \mathrm{ng} / \mathrm{mL})$ with normal parathyroid hormone $(55 \mathrm{pg} / \mathrm{mL})$ and serum total calcium levels $(9.8 \mathrm{mg} / \mathrm{dL})$. Other laboratory findings including complete blood count, erythrocyte sedimentation rate, C-reactive protein, electrolytes, alkaline phosphatase, thyroid function tests, and kidney and liver function tests were normal. Based on these findings, we thought that the cause of the knee pain was a stress fracture and dual-energy
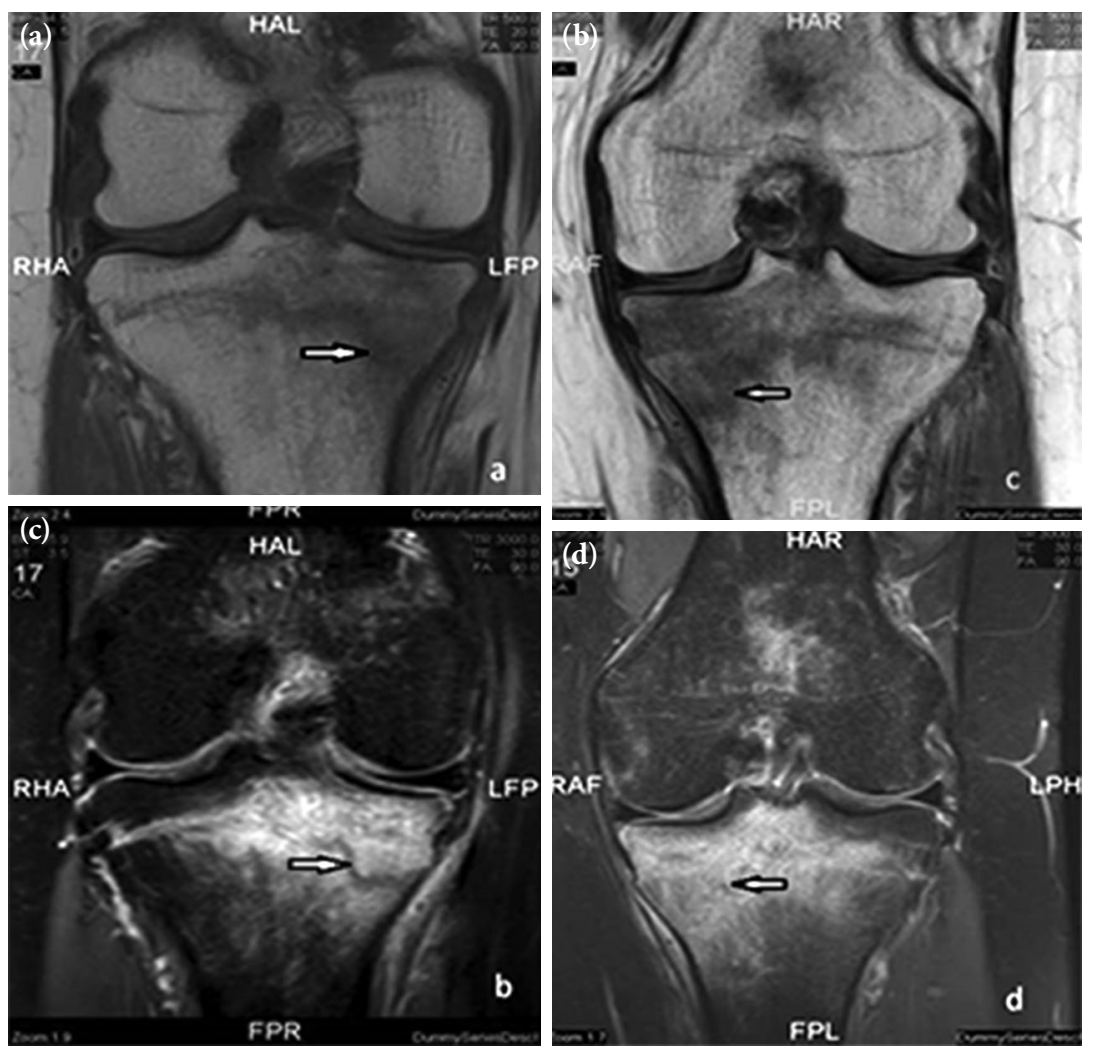

Figure 1. (a) Coronal turbo spin echo T1 and (b) spectral attenuated inversion recovery T2 (c) on the right knee, coronal turbo spin echo T1 and (d) spectral attenuated inversion recovery $\mathrm{T} 2$ on the left knee. Tibia metaphyseal stress fractures are seen in both knees. 
X-ray absorptiometry (DXA) was requested to rule out possible underlying osteoporosis. The DXA results were compatible with osteopenia in the lumbar region (total T-score of lumbar vertebrae: -1.7 , total Z-score: -1.6; total T-score of femur: 0.4, total Z-score: 0.4 ). The patient was diagnosed with pregnancy-related osteopenia and stress fracture of bilateral proximal tibia. Treatment included the cessation of weight bearing, resting, and supplementation with calcium and vitamin D 50,000 IU/week for four weeks and $50,000 \mathrm{IU} / \mathrm{month}$ for two months.

At four months of follow-up, the patient was asymptomatic and MRI of both knees showed significantly decreased bone marrow edema compared to the previous imaging and an incomplete fracture line at the medial sides of bilateral proximal tibia. Partial rupture of the anterior cruciate ligaments was

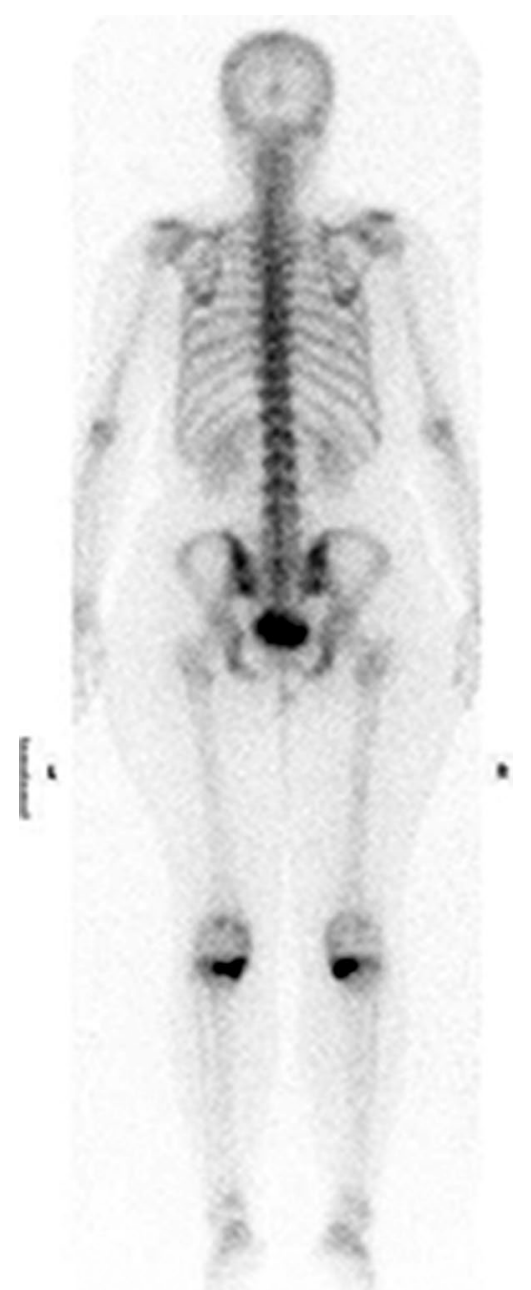

Figure 2. A scintigraphic view showing increased activity at bilateral proximal tibia. preserved (Figure 3). On physical examination, the patient was comfortable during the range of motion with no sign of suffering. The tests for meniscus and other ligamentous injury also yielded negative results. Vitamin D level was found to be $36 \mathrm{ng} / \mathrm{mL}$ after supplementation. During follow-up, the patient had no complaint about any symptom and the patient was scheduled for follow-up one year later. She was also informed about mobilization and weight bearing that should be increased gradually. A written informed consent was obtained from the patient.

\section{DISCUSSION}

Stress fractures mostly occur in athletes and military recruits, but they may also develop in the postpartum period. There are several case reports presenting postpartum stress fractures, particularly in the sacrum, pubic bone, and femur..$^{[8-10,12]}$ In the literature, only one case of unilateral tibial involvement has been reported to date. ${ }^{[7]}$ However, there are no other cases of postpartum stress fractures in bilateral tibia as we presented in this case report. Recognizing the disease and correction of the underlying and modifiable factors including osteopenia/osteoporosis, vitamin $\mathrm{D}$ deficiency, excessive intensity, and duration of exercise, the prognosis would be more favorable.

Vitamin D deficiency and low levels of calcium are the risk factors for development of stress fractures. A prospective, case-control study with female United States Navy recruits found that women in the lowest quintile of $25(\mathrm{OH}) \mathrm{D}$ serum concentrations (1.5 to $19.7 \mathrm{ng} / \mathrm{mL}$ ) had twice the risk of stress fracture compared to women in the highest quintile (over $40 \mathrm{ng} / \mathrm{mL}$ ). ${ }^{[13]}$ This study demonstrates that $30 \mathrm{ng} / \mathrm{dL}$, which is the usually accepted limit of vitamin D sufficiency, may be insufficient to minimize the stress fracture risk. In addition, some case-control studies showed that lower calcium intake was also associated with an increase in the stress fracture incidence in women, with a relative risk of 1.1 per $100 \mathrm{mg}$ decreased intake. ${ }^{[14,15]}$ During the pregnancy and lactation period, maternal calcium stores decrease due to the large amount of calcium transfer to the fetus or neonate and the calcium need is supplied by the release of calcium from maternal bone, increased intestinal absorption, or decreased renal excretion. ${ }^{[16]}$ It is also demonstrated that, as a consequence of the bone resorption, the bone turnover markers are increased during pregnancy. Therefore, adequate calcium consumption is crucial for pregnancy and postpartum period to prevent the development of complications. ${ }^{[17]}$ In our case, the 

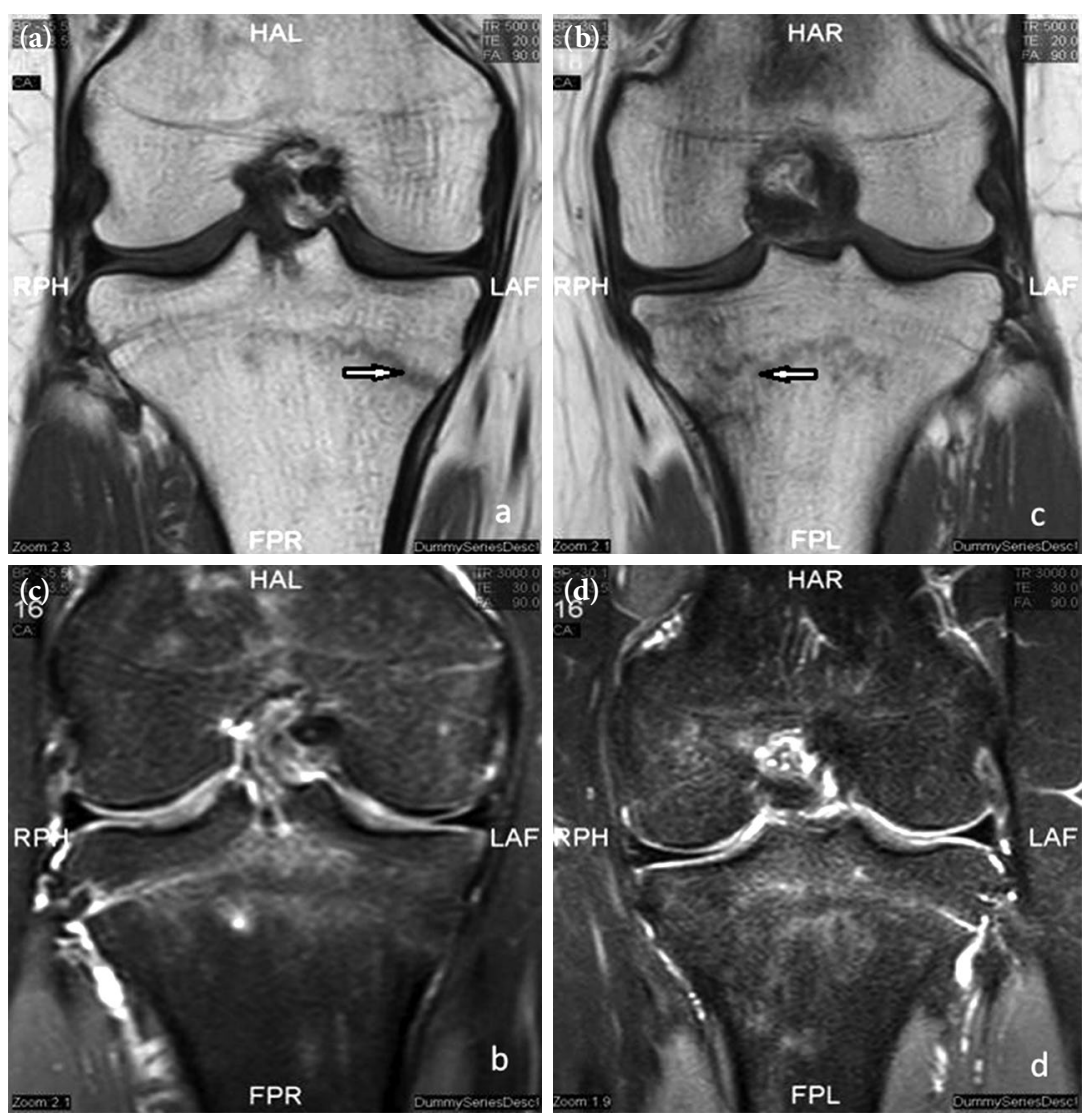

Figure 3. (a) Coronal turbo spin echo $\mathrm{T} 1$ and (b) spectral attenuated inversion recovery $\mathrm{T} 2$ on the right knee, (c) Coronal turbo spin echo $\mathrm{T} 1$ and (d) spectral attenuated inversion recovery $\mathrm{T} 2$ on the left knee. Control magnetic resonance imaging showing a reduction of bone marrow edema around fracture in both knees.

blood calcium level was normal, while vitamin D level was low and bone mineral densitometry revealed osteopenia. The patient did not pay attention to calcium consumption and did not use regular supplements. She was also sedentary during the pregnancy and, at the beginning of the lactation period, bilateral tibial stress fractures developed shortly after she started 2-h daily walks. Weight-bearing bones, particularly the lower extremity, are at the greatest risk for stress fractures. Tibial fractures account for $49 \%$ of all stress fractures seen in athletes, followed by fractures of the metatarsals. ${ }^{[18]}$ Greater than $12 \mathrm{~h}$ of exercise per week is also a risk factor for bone stress injury in women. ${ }^{[19]}$

When stress injury is suspected, the initially recommended modality is plain radiography. Linear sclerosis, periosteal reaction, cortical thickening or fracture lines may be seen; however, abnormal findings cannot be detected on plain radiographs. In such cases, MRI is preferred over CT and bone scintigraphy. It is the most sensitive modality with the findings including periosteal and bone marrow edema, intracortical signal abnormality, and visible fracture line extending from the cortex to the medullary canal. ${ }^{[1]}$ In our case, although radiographic findings were normal, MRI demonstrated an incomplete fracture line at the medial proximal tibia with bone marrow edema. We also used MRI during follow-up and the clinical improvement was found to be correlated with the improvement in MRI findings.

In conclusion, pain starting shortly after a vigorous physical activity should alert the physician to consider further investigations to find out the existence of bone lesions. The possibility of accompanying vitamin $\mathrm{D}$ deficiency or osteopenia, particularly in the postpartum period, should be also kept in mind in patients with a diagnosis of bone lesions such as stress fractures. Long term follow-up, resting, and accompanying symptomatic medication are an ideal treatment plan for the management of stress fractures. 


\section{Declaration of conflicting interests}

The authors declared no conflicts of interest with respect to the authorship and/or publication of this article.

\section{Funding}

The authors received no financial support for the research and/or authorship of this article.

\section{REFERENCES}

1. Matcuk GR Jr, Mahanty SR, Skalski MR, Patel DB, White EA, Gottsegen CJ. Stress fractures: pathophysiology, clinical presentation, imaging features, and treatment options. Emerg Radiol 2016;23:365-75.

2. Abbott A, Bird ML, Wild E, Brown SM, Stewart G, Mulcahey MK. Part I: epidemiology and risk factors for stress fractures in female athletes. Phys Sportsmed 2020;48:17-24.

3. Bennell KL, Brukner PD. Epidemiology and site specificity of stress fractures. Clin Sports Med 1997;16:179-96.

4. Pavlov H, Nelson TL, Warren RF, Torg JS, Burstein AH. Stress fractures of the pubic ramus. A report of twelve cases. J Bone Joint Surg [Am] 1982;64:1020-5.

5. Knapik J, Montain SJ, McGraw S, Grier T, Ely M, Jones BH. Stress fracture risk factors in basic combat training. Int J Sports Med 2012;33:940-6.

6. Bennell K, Matheson G, Meeuwisse W, Brukner P. Risk factors for stress fractures. Sports Med 1999;28:91-122.

7. Clemetson IA, Popp A, Lippuner K, Ballmer F, Anderson SE. Postpartum osteoporosis associated with proximal tibial stress fracture. Skeletal Radiol 2004;33:96-8.

8. Yan CX, Vautour L, Martin MH. Postpartum sacral insufficiency fractures. Skeletal Radiol 2016;45:413-7.

9. Mikawa Y, Watanabe R, Yamano Y, Miyake S. Stress fracture of the body of pubis in a pregnant woman. Case report. Arch Orthop Trauma Surg 1988;107:193-4.

10. Billey T, Dromer C, Pagès $M$, Caulier $M$, Lassoued $S$,
Fournié B. Spontaneous fracture of the femoral neck in hip algodystrophy in pregnancy. Apropos of a case and review of the literature. Rev Rhum Mal Osteoartic 1992;59:494-6.

11. Pouwels S, Dabekausen YA. Rib stress fractures in pregnancy: a case report and review of literature. Clin Exp Obstet Gynecol 2017;44:296-8.

12. Pegrum J, Crisp T, Padhiar N. Diagnosis and management of bone stress injuries of the lower limb in athletes. BMJ 2012;344:e2511.

13. Burgi AA, Gorham ED, Garland CF, Mohr SB, Garland FC, Zeng K, et al. High serum 25-hydroxyvitamin D is associated with a low incidence of stress fractures. J Bone Miner Res 2011;26:2371-7.

14. Myburgh KH, Hutchins J, Fataar AB, Hough SF, Noakes TD. Low bone density is an etiologic factor for stress fractures in athletes. Ann Intern Med 1990;113:754-9.

15. Cobb KL, Bachrach LK, Sowers M, Nieves J, Greendale GA, Kent KK, et al. The effect of oral contraceptives on bone mass and stress fractures in female runners. Med Sci Sports Exerc 2007;39:1464-73.

16. Prentice A, Jarjou LM, Cole TJ, Stirling DM, Dibba B, Fairweather-Tait S. Calcium requirements of lactating Gambian mothers: effects of a calcium supplement on breastmilk calcium concentration, maternal bone mineral content, and urinary calcium excretion. Am J Clin Nutr 1995;62:58-67.

17. Thomas M, Weisman SM. Calcium supplementation during pregnancy and lactation: effects on the mother and the fetus. Am J Obstet Gynecol 2006;194:937-45.

18. Matheson GO, Clement DB, McKenzie DC, Taunton JE, Lloyd-Smith DR, MacIntyre JG. Stress fractures in athletes. A study of 320 cases. Am J Sports Med 1987;15:46-58.

19. Barrack MT, Gibbs JC, De Souza MJ, Williams NI, Nichols JF, Rauh MJ, et al. Higher incidence of bone stress injuries with increasing female athlete triad-related risk factors: a prospective multisite study of exercising girls and women. Am J Sports Med 2014;42:949-58. 